\title{
Die Methode möglicher Fälle und die Methode typischer Fälle: Frank Jackson vs. Oswald Hanfling
}

\section{$1 \quad$ Einleitung}

Im vorangehenden Kapitel habe ich mich unter anderem damit auseinandergesetzt, welche Strategien zur (konnektiven) Analyse eines Begriffs bzw. zur Erlangung eines Überblicks über die etablierten Regeln zur Verwendung eines sprachlichen Ausdrucks Wittgenstein vorschlägt. Einer der Ansatzpunkte bestand darin, dass wir uns mit konkreten, einzelnen Situationen der Verwendung eines Ausdrucks auseinandersetzen sollten und versuchen sollten, uns darüber klar zu werden, was der Ausdruck in diesen Situationen leistet. Ein gutes Beispiel für einen vom späten Wittgenstein beeinflussten Philosophen, der sich diese Methode zu eigen gemacht hat, ist Oswald Hanfling, dessen Vorgehen bei der Analyse des Wissensbegriffs im vorliegenden Kapitel skizziert werden soll. Diese Betrachtung dient zweierlei Zwecken: Zum einen dient sie der Illustration der im vorliegenden Buch verteidigten Art und Weise, Philosophie zu betreiben. Zum anderen soll später beurteilt werden, ob es sich bei der von Hanfling verwendeten und in der vorliegenden Abhandlung angepriesenen Strategie zur Auflösung philosophischer Probleme um die so genannte Methode möglicher Fälle handelt, die in der Literatur bisweilen als die Methode der Begriffsanalyse deklariert wird. ${ }^{1}$

Die Bezeichnung »method of possible cases « stammt von Frank Jackson, der gleichzeitig ihr prominentester Anhänger ist. Im Abschnitt 2 des vorliegenden Kapitels werden Jacksons Ausführungen zur Methode möglicher Fälle rekonstruiert und Hanflings Vorgehen bei der Analyse des Wissensbegriffs gegenübergestellt. Im Abschnitt 3 wird ein Einwand gegen Jacksons Auffassung der Methode möglicher Fälle rekonstruiert und evaluiert.

Wie man sich die Klärung eines philosophisch interessanten Begriffs im Sinne der im Kapitel 1 charakterisierten Philosophie-Konzeption konkret

1 Vgl. z. B. Nimtz 2012, S. 221, Jackson 1998, S. 38, 42. 
vorzustellen hat, lässt sich anhand von Oswald Hanflings Analyse des Wissensbegriffs in Philosophy and Ordinary Language illustrieren. ${ }^{2}$ Es ist anzunehmen, dass Hanfling bei dieser Analyse ungefähr wie folgt vorgegangen ist: Er hat sich zunächst einige spezifische Situationen vergegenwärtigt, in welchen es natürlich wäre, den Ausdruck »wissen« zu verwenden:

(1) Ich klopfe an die Tür des Büros, in dem meine Kollegen arbeiten, und frage sie: »Weiß jemand von euch, wo Sabine ist?«.

(2) Ich frage jemanden: »In welchem Raum findet das Seminar morgen statt? «, und die andere Person entgegnet: »Ich weiß es nicht«.

(3) Ich kritisiere meinen Mitbewohner dafür, dass er mich mit lauter Musik geweckt hat, und er verteidigt sich: »Ich wusste nicht, dass du noch schliefst.«

(4) Ich gebe Auskunft über die Gästeliste für mein Geburtstagsfest und erwähne unter anderem: »Meinen Bruder habe ich nicht eingeladen, weil ich weiß, dass er dann in den Ferien ist.«

(5) Mein Kollege teilt mir mit:»Arsenal ist schon wieder im Achtelfinale ausgeschieden!«, und ich entgegne: »Ich weiß.«

Dann hat Hanfling diese Situationen klassifiziert. In Situationen wie (1) und (2), so Hanfling, geht es um Wissen als eine Fähigkeit - die Fähigkeit, jemanden zu informieren -, während es in Fällen wie (3), (4) und (5) um Wissen in dem Sinne geht, dass sich jemand einer Tatsache bewusst ist. ${ }^{3}$ Der Zweck der Verwendung des Verbs »wissen $«$ unterscheidet sich in den verschiedenen Situationen. In Situationen der Art von (1) und (2) wird »wissen« zur Identifikation von potentiellen Informantinnen oder Informanten gebraucht (resp. in (2), um sich selbst als Informantin oder Informanten auszuschließen). Hanfling nennt Situationen dieser Art »enquiring situations«. Demgegenüber geht es in (3) und (4) um die Begründung von Verhaltensweisen. Situationen dieser Art bezeichnet Hanfling als »commenting situations«. Der Situationstyp (5) schließlich ist hinsichtlich seiner Funktion ein Spezialfall, dem Hanfling den Namen »no-need-to-tell situation « gibt. ${ }^{4}$ Diese drei Situationstypen sind laut Hanfling für die Bedeutung von »wissen « zentral, auch wenn er einräumt, dass es auch Situationen gibt, in welchen der Verwendung des Wortes »wissen « eine andere Funktion zukommt - etwa die, einen besonderen Grad von Sicherheit auszudrücken: »Ich glaube nicht nur, dass $p$, sondern ich weiß es. « ${ }^{5}$

2 Hanfling 2000, Kap. 6.

3 Vgl. Hanfling 2000, S. 96f. Das englische Wort, dass Hanfling im Zusammenhang mit Situationen wie (3) oder (4) verwendet, ist »awareness«.

4 Vgl. Hanfling 2000, S. $96 f$.

5 Vgl. Hanfling 2000, S. 96, 98, 104f. 
Sofern Hanfling bei seiner Analyse des Wissensbegriffs so vorgegangen ist wie oben vorgeschlagen, spielen mögliche Fälle des Gebrauchs des Wortes »wissen « für seine Untersuchung eine wichtige heuristische Rolle. Hat Frank Jackson etwas Ähnliches wie Hanflings Vorgehensweise im Sinn, wenn er in From Metaphysics to Ethics von der »method of possible cases «, ${ }^{6}$ der Methode möglicher Fälle spricht?

An der Stelle, an welcher Jackson die Methode möglicher Fälle erstmals erwähnt, ist von der Methode als Mittel zur Überprüfung der Analyse eines Begriffs die Rede - davon, dass eine adäquate Begriffsanalyse der Methode möglicher Fälle standhalten muss: Die Analyse von Wissen als wahre, gerechtfertigte Überzeugung kann nur adäquat sein, wenn wir keinen möglichen Fall von Wissen finden können, der nicht gleichzeitig ein Fall von wahrer, gerechtfertigter Überzeugung ist - und umgekehrt auch keinen Fall von wahrer, gerechtfertigter Überzeugung, der nicht gleichzeitig ein Fall von Wissen ist. Edmund Gettiers Argument in seinem berühmten Artikel »Is Justified True Belief Knowledge? « (1963) kann in diesem Sinn als Anwendung der Methode möglicher Fälle aufgefasst werden. Auch in »Thought Experiments and Possibilities « (2009) beschreibt Jackson den Nutzen der Methode möglicher Fälle zunächst so, dass der Eindruck entsteht, die Methode diene insbesondere dazu, bereits formulierte Hypothesen über die Kriterien für das Fallen unter einen Ausdruck (aber auch beispielsweise über Kriterien für moralische Zulässigkeit) durch ihre Anwendung auf spezielle Fälle zu testen oder zu erläutern. ${ }^{7}$ Die Methode möglicher Fälle scheint Jackson zufolge nicht in erster Linie eine Methode dafür zu sein, erst einmal zu Hypothesen - beispielsweise über die Anwendbarkeitsbedingungen eines sprachlichen Ausdrucks - zu gelangen. An anderen Stellen wird jedoch deutlich, dass Jackson zufolge der Methode möglicher Fälle auch diese Funktion zukommt. So schreibt er etwa:

But how should we identify our ordinary conception [of free action or intentional states]? The only possible answer, I think, is by appeal to what seems to us most obvious and central about free action, determinism, belief, or whatever, as revealed by our intuitions about possible cases. [...] For what guides me in describing an action as free is revealed by my intuitions about whether various possible cases are or are not cases of free action. Thus my intuitions about possible cases reveal my theory of free action [...]. To the extent that [my] intuitions coincide with those of the folk, they reveal the folk theory. ${ }^{8}$

6 Z. B. Jackson 1998, S. 28.

7 Vgl. Jackson 2009, S. 10of.

8 Jackson 1998, S. 31f. 
Jacksons Idee ist die folgende: Wer einen Begriff besitzt, ist in der Regel dazu in der Lage, zu beurteilen, ob ein bestimmter Fall zur Extension des Begriffs gehört oder nicht. ${ }^{9}$ Welches unser Begriff des freien Willens ist, können wir herausfinden, indem wir eine Reihe von echten und konstruierten Situationen beschreiben, in welchen eine Person eine Handlung ausführt, und diese Situationen dahingehend beurteilen, ob wir jeweils die Intuition haben, dass die Handlung aus freiem Willen erfolgt ist oder nicht. Die Handlungsbeschreibung muss zu diesem Zweck zwar alle Informationen enthalten, die für die Entscheidung relevant sind, ob es sich um einen Fall von freiem Willen handelt oder nicht; sie darf aber natürlich nicht den Ausdruck »freier Wille« oder damit verwandte Ausdrücke enthalten. ${ }^{10}$

Mit »Intuitionen « sind in diesem Kontext keine spontanen Eingebungen gemeint; es geht um wohlüberlegte Urteile darüber, ob eine Handlung unter den Begriff des freien Willens fällt oder nicht. ${ }^{11}$ Mit der Zeit sind wir aufgrund der Betrachtung unserer Intuitionen in Bezug auf eine Reihe von Fällen dazu in der Lage, eine Hypothese darüber aufzustellen, welche Aspekte der Fälle, in welchen wir freien Willen zuschreiben, dafür ausschlaggebend sind, dass sie unter unseren Begriff des freien Willens fallen: Wir erkennen ein bestimmtes Muster oder eine Regelmäßigkeit in den Fällen, die wir als zur Extension des Begriffs des freien Willens gehörig identifiziert haben. ${ }^{12}$ Diese Hypothese wird dann hinsichtlich ihrer Übereinstimmung mit unserer Beurteilung weiterer möglicher Fälle überprüft und gegebenenfalls modifiziert, bis wir keine Gegenbeispiele mehr zu unserer Hypothese finden können.

Das Ziel des ganzen Verfahrens besteht im Grunde genommen darin, einen sprachlichen Ausdruck $a_{2}$ zu finden, der genau dann auf einen Fall Anwendung findet, wenn der Ausdruck $a_{p}$, dessen Bedeutung zu klären ist, auf ihn Anwendung findet. ${ }^{13}$ Im Falle von »wissen « dachten vor Gettier viele Leute, dass »wahre, gerechtfertigte Überzeugung « dieses Kriterium erfüllt. ${ }^{14}$ Anhand von Jacksons Beschreibung dieses Beispiels einer (missglückten) Anwendung der Methode möglicher Fälle vonseiten der Epistemologie vor Gettier kommt

9 Vgl. Chalmers/Jackson 2001, S. $322 f$.

10 Vgl. Chalmers/Jackson 2001, S. 323. Chalmers und Jackson sprechen in diesem Zusammenhang von »nontrivially sufficient information« (Kursivierung im Original).

11 Vgl. Jackson 1998, S. 35.

12 Vgl. Jackson 2011, S. 474, Chalmers/Jackson 2001, S. 322.

13 Vgl. Chalmers/Jackson 2001, S. 322f. Ob $a_{2}$ dann dieselbe Bedeutung wie $a_{1}$ hat, muss in diesem Stadium der Analyse allerdings noch offen gelassen werden. Jackson betont an verschiedener Stelle, dass notwendige Koextensionalität nicht hinreichend für Bedeutungsidentität ist. Vgl. z. B. Jackson 1998, S. 34.

14 Gettier selbst führt Platon, Roderick Chisholm und A. J. Ayer als Beispiele an. Vgl. Gettier 1963, S. 121. 
ein wichtiger Aspekt seiner Auffassung der Begriffsanalyse zum Ausdruck, der über die Anwendung der Methode möglicher Fälle hinausgeht:

How [...] did the supporters of the true justified belief analysis of knowledge arrive at that analysis? They took examples they recognised as cases of knowledge. They looked for the common pattern in the examples. As most ordinary cases of knowledge are cases of true justified belief, and most ordinary cases of true justified belief are cases of knowledge, they (understandably) thought that the right way to articulate the pattern they recognised was to use the words 'true justified belief'. In addition and importantly, they paid attention to the theoretical purposes we philosophers put the notion of knowledge to in epistemology, and judged that true justified belief served those purposes. ${ }^{15}$

Eine adäquate Analyse eines Begriffs muss Jackson zufolge auch der Rolle gerecht werden, die der betreffende Begriff in unseren Theorien spielt. In diesem Zusammenhang müssen bei Jackson zwei Aspekte unterschieden werden: Das Verhältnis zwischen Begriffen und so genannten »Alltagstheorien « (»folk theories«) und das Verhältnis zwischen Begriffen und wissenschaftlichen Theorien.

Zunächst zu den Alltagstheorien: An vielen Stellen, an welchen bei Jackson beispielsweise von der Alltagstheorie, die wir von Überzeugungen, freiem Willen oder Wissen haben, die Rede ist, scheint er eigentlich auf unseren $B e$ griff der Überzeugung, des freien Willens oder des Wissens Bezug zu nehmen: Indem wir unseren Begriff des freien Willens beschreiben respektive indem wir beschreiben, was dafür ausschlaggebend ist, dass wir über eine Handlung sagen würden, dass sie aus freiem Willen ausgeführt wird, beschreiben wir das, was Jackson die »Alltagstheorie« des freien Willens nennt. ${ }^{16}$ An anderen Stellen klingt die Idee an, dass unsere Alltagstheorien Hypothesen umfassen, die prinzipiell widerlegbar sind. So schreibt Jackson zum Beispiel:

We have $[\ldots]$ a folk theory that ties together understanding, truth, and information about possibilities; and the obvious way to articulate this folk theory is to identify, or at least essentially connect, understanding a sentence with knowing the conditions under which it is true [...]. This kind of theory in its philosophically sophisticated articulations is best known through the work of David Lewis and Robert Stalnaker. But it would, I think, be wrong to regard the folk theory as being as controversial as these articulations. The folk theory is, it seems to me, a commonplace. ${ }^{17}$

\footnotetext{
15 Jackson 2011, S. 474.

16 Vgl. etwa Jackson 1998, S. 31f., $38 f$.

17 Jackson 1998, S. 71.
} 
Es ist anzunehmen, dass Jackson der Meinung ist, dass Begriffe oder unser Begriffssystem Hypothesen beinhalten können und dass deshalb die beiden oben genannten Ideen - die Auffassung, dass Alltagstheorien Begriffe sind, und die Auffassung, dass sie Hypothesen umfassen - in seinen Augen miteinander verträglich sind. Auf diesen Punkt werde ich weiter unten im Zusammenhang mit der Abgrenzung von Jackson gegenüber Hanfling zurückkommen.

Neben unseren Alltagstheorien gibt es Jackson zufolge, wie bereits erwähnt, auch andere Theorien, die mit unseren Begriffen zusammenhängen, nämlich die Theorien, die in den Wissenschaften aufgestellt werden. Einige unserer Begriffe sind derart mit wissenschaftlichen Theorien verknüpft, so Jacksons Auffassung, dass ein wissenschaftlicher Fortschritt in der Erkenntnis resultieren kann, dass wir die betreffenden Ausdrücke bisher falsch verwendet haben. Ein Beispiel hierfür, das Jackson in From Metaphysics to Ethics anspricht, ist der Begriff Fisch. Dieser umfasst Jackson zufolge neben bestimmten Oberflächenmerkmalen die Bedingung, dass die Tiere, die unter ihn fallen, gemäß der besten wissenschaftlichen Theorie zu ein und derselben Art gehören. Die Einsicht, dass der Besitz von Kiemen ein wichtigeres Unterscheidungsmerkmal ist als das Leben unter Wasser, hat mithin dazu geführt, dass die Menschen erkannt haben, dass Wale und Delfine entgegen ihrer früheren Annahme nie zur Extension von »Fisch « gehört haben. ${ }^{18}$ In anderen Fällen sehen wir uns Jackson zufolge im Lichte bestimmter wissenschaftlicher oder philosophischer Fortschritte dazu veranlasst, unsere Begriffe zu revidieren - insbesondere deshalb, weil ein anderer Begriff, zu dem unser aktueller Begriff als eine Annäherung verstanden werden kann, sich als nützlicher herausstellt. ${ }^{19}$

Welches ist nun die Rolle, die der Begriff des Wissens in unseren Theorien spielt? Jackson zufolge ist es die Rolle des epistemischen Goldstandards: Wissen ist epistemisch das Beste, was wir erlangen können; wir erreichen Wissen genau dann, wenn auf dem Weg zu einer Überzeugung alles richtig abgelaufen ist. Gettier hat gezeigt, dass wir zu wahrer, gerechtfertigter Überzeugung auch per Zufall gelangen können, wenn im Grunde genommen epistemisch etwas schiefläuft. Jackson zieht aus dieser Erkenntnis von Gettier in verschiedenen Publikationen unterschiedliche Schlussfolgerungen: In From Metaphysics to Ethics schreibt er, Gettier habe gezeigt, dass unser Begriff des Wissens entgegen der zeitweise etablierten Meinung nie mit unserem Begriff von wahrer,

18 Vgl. Jackson 1998, S. 35. Welche Unterscheidungsmerkmale wichtiger sind als andere, hängt dabei vom Zweck der Unterscheidung ab. Für die Zwecke des Fangens und der Zucht von Tieren können andere Merkmale wichtig sein als für den Zweck einer biologischen Klassifikation.

Vgl. Jackson 1998, S. 44f., Chalmers/Jackson 2001, S. 323, Fußnote 6. 
gerechtfertigter Überzeugung identisch gewesen sei. ${ }^{20}$ Demgegenüber schreibt er in »Thought Experiments and Possibilities«, er könne nicht ausschließen, dass sein Wissensbegriff durch die Lektüre von Gettiers »Is Justified True Belief Knowledge?« revidiert worden sei (und vorher mit seinem Begriff von wahrer, gerechtfertigter Überzeugung übereingestimmt habe). ${ }^{21}$

Es gibt auch Begriffe, für deren Analyse die Methode möglicher Fälle nicht geeignet ist, so Jackson, der beispielsweise in »Conceptual Analysis and Reductive Explanation « zusammen mit David Chalmers geltend macht, dass wir den Begriff der Zeit nicht klären können, indem wir unsere Anwendung von temporalen Begriffen auf Situationen prüfen, die ohne Einbezug solcher Begriffe beschrieben werden. Analoges gilt laut Jackson und Chalmers eventuell für die Begriffe des Raums, der Ursache und des Bewusstseins. ${ }^{22}$ Die Anwendung der Methode möglicher Fälle ist Jackson zufolge auch kein hilfreicher Ansatzpunkt für die Beantwortung der Frage, ob Kontradiktionen alles implizieren. Eine Philosophin, die sich mit dieser Frage auseinandersetzt, sollte, so Jackson, nicht versuchen, Beispiele von Kontradiktionen dahingehend zu untersuchen, ob sie über sie sagen würde, dass sie alles implizieren, oder nicht, sondern sich über die Rolle klarzuwerden versuchen, die Implikationen in unseren Theorien spielen, und überprüfen, ob wir angesichts dieser Rolle dazu gezwungen sind, zu sagen, dass Kontradiktionen in einem Implikationsverhältnis zu sämtlichen Urteilen stehen. ${ }^{23}$

Wenn wir vor dem Hintergrund dieser Rekonstruktion von Jacksons Auffassung der Methode möglicher Fälle und der Rolle, die ihr in der Philosophie zukommt, noch einmal Hanflings oben beschriebene Analyse des Wissensbegriffs betrachten, springt zunächst einmal der folgende Unterschied zwischen Jacksons und Hanflings Methoden ins Auge: Während Jackson verschiedene Fälle in Bezug darauf beurteilt, ob sie unter einen bestimmten Begriff - zum Beispiel den Begriff des Wissens - fallen, und auf dieser Grundlage versucht, die Kriterien für das Fallen unter den Begriff zu bestimmen, wählt Hanfling direkt Fälle aus, die charakteristisch für die Verwendung von »wissen« sind, und befasst sich dann mit der Frage, was das Wort in den betreffenden

$20 \quad$ Vgl. Jackson 1998, S. 36.

21 Vgl. Jackson 2009, S. 103. Diese Möglichkeit wird auch in Jackson 2010, S. 184 und Jackson 2011, S. 475, Fußnote 7 in Erwägung gezogen. Die Idee, dass unser Wissensbegriff vor Gettier dem Begriff von wahrer, gerechtfertigter Überzeugung entsprochen hat, scheint allerdings nur dann haltbar zu sein, wenn wir nicht darauf bestehen, dass es schon immer Teil unseres Wissensbegriffs war, dass Wissen die Rolle des epistemischen Goldstandards spielt.

22 Vgl. Chalmers/Jackson 2001, S. 325.

23 Vgl. Jackson 1998, S. 36. 
Kontexten leistet. Man könnte Hanflings Methode in Abgrenzung zu Jacksons Methode möglicher Fälle deshalb als »Methode typischer Fälle« bezeichnen. ${ }^{24}$ Bei Jackson stellen wir auf der Grundlage der Betrachtung möglicher Fälle Hypothesen darüber auf, unter welchen Bedingungen ein Akteur über Wissen verfügt, und überprüfen dann anhand von weiteren oder veränderten Beispielen, ob die Hypothese sich bewährt. Finden wir Gegenbeispiele zum formulierten Definitionsversuch, versuchen wir, eine neue, verbesserte Hypothese aufzustellen. Demgegenüber befasst sich Hanfling nicht mit der Suche nach notwendigen und hinreichenden Bedingungen für das Fallen unter den Begriff des Wissens, sondern bestimmt die zentralen Situationstypen, in welchen das Wort »wissen « verwendet wird, und beschreibt, welchem Zweck die Verwendung von »wissen « in diesen Situationen dient. Ein »Gegenbeispiel« zu seiner Analyse kann also höchstens ein Szenario sein, das typisch ist für die Verwendung von »wissen « und keinem der von ihm aufgezählten Typen untergeordnet werden kann. Wenn ein solches Beispiel gefunden wird, muss Hanfling seine Analyse nicht verwerfen, sondern um einen weiteren Situationstyp ergänzen. Eine andere mögliche Kritik an einer Analyse von der Art, wie Hanfling sie präsentiert, könnte im Versuch bestehen, zu zeigen, dass es eine bessere oder systematischere Weise gibt, die Beispiele zu klassifizieren. Dass beispielsweise zwei von Hanflings Situationstypen eigentlich Instanzen eines dritten, übergeordneten Situationstyps sind. In den nachfolgenden Abschnitten wird eine Reihe von weiteren Unterschieden zwischen Jacksons und Hanflings Methoden besprochen.

\section{Intuitionen}

Sowohl bei Jackson als auch bei anderen Philosophinnen und Philosophen, die seine Methode aufgreifen, findet sich regelmäßig die Bemerkung, man berufe sich auf »Intuitionen über mögliche Fälle«. Auf diesem Aspekt liegt auch ein Schwerpunkt in der weiter unten diskutierten kritischen Literatur zur Nützlichkeit der Methode möglicher Fälle. Jackson macht allerdings in »On Gettier Holdouts« geltend, dass in seinen Augen im Zusammenhang mit der Methode möglicher Fälle nicht viel Gewicht auf dem Begriff der Intuition liegt. Um beispielsweise zu überprüfen, ob seine Studierenden seine Intuitionen in Bezug auf Gettier-Fälle teilen, frage er sie, nachdem er einen Fall beschrieben habe, ob sie die entsprechenden Akteure so beurteilen würden, dass sie über Wissen verfügen, oder nicht. Er erkläre nicht, dass er sich für ihre Intuitionen

24 Vgl. dazu auch Ernst (2015, S. 184, 186), der die von ihm so genannte »Definitionsvorschlag- und Gegenbeispiel-Methode« der »Normalfall-Methode« gegenüberstellt und Letztere explizit unter anderem mit Hanfling assoziiert. 
interessiere, im Gegensatz etwa zu ihren Überzeugungen in Bezug auf die beschriebenen Fälle. Wenn er in einzelnen Befragungen trotzdem das Wort »Intuition « verwende, dann höchstens, um deutlich zu machen, dass er wissen wolle, was die eigenen Urteile der Studierenden seien, und sich nicht dafür interessiere, was sie vielleicht irgendwo gelesen haben oder für die Meinung von Epistemologinnen und Epistemologen halten. ${ }^{25}$

Im Gegensatz zu Jackson distanziert sich Hanfling vollständig vom Gebrauch von Intuitionen in der Philosophie. ${ }^{26} \mathrm{Ihm}$ zufolge interessieren wir uns in der Philosophie dafür, was wir sagen (würden). Und um beispielsweise festzustellen, dass ein Beispiel als ein Fall von Wissen zu beschreiben ist, müssen wir weder auf Intuitionen noch auf Meinungen oder Überzeugungen zurückgreifen, sondern können ganz einfach von unserer Sprachkompetenz Gebrauch machen. ${ }^{27}$

\section{Empirie}

Jackson fasst die Analyse von Begriffen als empirisches Unterfangen auf. In From Metaphysics to Ethics vergleicht er die Anwendung der Methode möglicher Fälle zur Klärung philosophisch relevanter Begriffe mit der Vorgehensweise von Kinder-Psychologen, die herausfinden wollen, was Kinder unter »faster than « verstehen, ${ }^{28}$ oder von Soziologen, die untersuchen, welchen Begriff des Sozialisten Amerikaner - im Gegensatz etwa zu Briten oder Franzosen - haben. ${ }^{29}$ Auf der Grundlage dessen, wie Kinder, Amerikaner, Briten, Franzosen oder wir selbst bestimmte mögliche Fälle klassifizieren, finden wir heraus, welches ihre und unsere Begriffe sind. Die Begriffsanalyse ist aber Jackson zufolge immerhin in dem Sinne a priori, dass wir, um zu beurteilen, ob wir beispielsweise einem Akteur in einer bestimmten Situation Wissen zuschreiben würden oder nicht, nicht wissen müssen, ob die Situation echt oder konstruiert ist. Wir müssen, um festzustellen, unter welchen Bedingungen wir dazu bereit sind, den Ausdruck »wissen « zu verwenden, im Wesentlichen nicht wissen, was in der Welt tatsächlich der Fall ist. ${ }^{30}$ Christian Nimtz bezeichnet

25 Vgl. Jackson 2011, S. 471.

26 Vgl. Hanfling 2000, S. 241-243.

27 Vgl. z. B. Hanfling 2000, S. 54, 58.

28 Offenbar sagen Kinder in einem bestimmten Alter genau dann, dass ein Gegenstand $a$ schneller ist als ein Gegenstand $b$, wenn $a$ früher als $b$ ein vorgegebenes Ziel erreicht unabhängig davon, an welchen Punkten $a$ und $b$ gestartet sind. Vgl. Jackson 1998, S. $32 f$.

Vgl. Jackson 1998, S. 32f., 47 .

3o Vgl. Jackson 1998, S. 51. Nimtz, dessen Auffassung der Begriffsanalyse sich ansonsten sehr nahe an derjenigen von Jackson befindet, schreibt in »Begriffsanalyse heute«, bei der Begriffsanalyse handle es sich nur dann um ein empirisches Projekt, wenn wir herausfinden wollen, wie »wir« oder eine ganze Sprachgemeinschaft einen Ausdruck (der kein Name 
diesen Wesenszug der Begriffsanalyse als schwache Apriorität.$^{31}$ Einen Spezialfall stellen in diesem Zusammenhang Jackson (und auch Nimtz) zufolge die Namen natürlicher Arten dar. Um herauszufinden, worauf sich beispielsweise das Wort »Wasser « in kontrafaktischen Welten bezieht, sind - anders als im Fall von »wissen « - empirische Ergebnisse der Naturwissenschaften erforderlich: Erkenntnisse darüber, was in der Welt tatsächlich der Fall ist (worin zum Beispiel Wasser in unserer Welt besteht). Die Analyse von Begriffen wie Wasser ist also im Gegensatz zur Analyse von Begriffen wie Wissen nicht schwach a priori, sondern a posteriori. ${ }^{32}$

Hanfling zufolge müssen wir zur Analyse von Begriffen nicht auf empirische Methoden zurückgreifen, da eine solche Analyse nicht auf die Erlangung von neuem Wissen gerichtet ist, sondern auf die Kodifikation von Wissen, das wir bereits besitzen, beziehungsweise darauf, einen Überblick über die Art und Weise zu erlangen, auf welche wir einen bestimmten sprachlichen Ausdruck verwenden. ${ }^{33}$ Anders als Jackson fasst Hanfling Urteile über die Bedeutung unserer Wörter deshalb auch nicht als Hypothesen auf. ${ }^{34}$

\section{Begriffe}

Ich habe weiter oben bereits geschrieben, dass Hanflings Analyse des Wissensbegriffs nicht darauf gerichtet ist, notwendige und hinreichende Bedingungen für das Fallen unter den Begriff Wissen zu bestimmen. Dies liegt unter anderem daran, dass er mit Wittgenstein die Auffassung ablehnt, dass für jedes Prädikat ein Merkmal oder eine Menge von Merkmalen gefunden werden kann, die allen und nur den Entitäten zukommen, die zur Extension des betreffenden Begriffs gehören:

Another plausible assumption is that when we apply the same word to a range of different things, there must be something that they, and only they, have in common, in virtue of which that word is applicable. This idea, according to Wittgenstein, 'has shackled philosophical investigation', leading philosophers to 'dismiss

einer natürlichen Art ist) verwenden/verwendet. Zur Beschreibung der Art und Weise, wie ich selbst einen Ausdruck gebrauche, ist Nimtz zufolge keine empirische Untersuchung vonnöten (vgl. Nimtz 2012, S. 225).

31 Vgl. Nimtz 2012, S. 225f. Auf die Frage nach der Apriorität der Begriffsanalyse komme ich im Abschnitt 3 des vorliegenden Kapitels im Zusammenhang mit dem Einwand von Janice Dowell zurück.

Genauer gesagt, ist die Erkenntnis ihrer A-Intensionen schwach a priori, aber die Erkenntnis ihrer C-Intensionen a posteriori. Vgl. Jackson 1998, S. 46-52. Vgl. auch S. 103 des vorliegenden Kapitels.

33 Vgl. Hanfling 2000, S. 57 f.

34 Vgl. z. B. Jackson 1998, S. 36. 
as irrelevant the concrete cases, which alone could have helped [them] to understand the usage' of such words as 'knowledge' and others (BB 19-20). ${ }^{35}$

In seiner Analyse des Wissensbegriffs liegt Hanflings Fokus denn auch weniger darauf, welche Merkmale dafür ausschlaggebend sind, dass in den einzelnen Fällen Wissen vorliegt oder nicht vorliegt, sondern darauf, welches die (kommunikative) Funktion oder der Witz $^{36}$ der Verwendung des Wortes »wissen" in den einzelnen Situationen ist. ${ }^{37}$ Demgegenüber kommt bei Jackson immer wieder die Annahme zum Ausdruck, dass die verschiedenen Fälle, auf welche die Begriffe der Willensfreiheit, der Überzeugung und eben auch des Wissens Anwendung finden, jeweils ein gemeinsames Muster (pattern) aufweisen, welches wir im Zuge der Betrachtung der Extension des betreffenden Begriffs über mögliche Fälle hinweg erkennen können. ${ }^{38}$ Die Einteilung von Beispiel-Situationen in Fälle, die unter den zu analysierenden Begriff fallen, und solchen, die nicht unter ihn fallen, ist für Jacksons Methode deshalb auch von viel größerer Bedeutung als für Hanfling. Gelegentlich macht es den Anschein, als würde Jackson Begriffe mit (Funktionen auf) Extensionen über mögliche Welten hinweg identifizieren, mit der Qualifikation, dass bei der Identifikation komplexer Begriffe auch die Extensionen ihrer Teil-Begriffe zu berücksichtigen sind, so dass dem Umstand Rechnung getragen werden kann, dass zum Beispiel »gleichwinkliges Dreieck« und »gleichseitiges Dreieck « nicht denselben Begriff ausdrücken. ${ }^{39}$ In anderen Passagen ist Jackson vorsichtiger und schreibt beispielsweise:

Surely part of the answer [to the question as to what we use 'knowledge' for] lies in what a user of the word says about its application or non-application to various possible cases. It follows that part (part) of what is going on when we appeal to intuitions about possible cases is an investigation into what people

35 Hanfling 200o, S. 4of. Das eingefügte »them« stammt von Hanfling.

36 Vgl. zum Begriff des Witzes auch das Kapitel 3 des vorliegenden Buchs sowie Ertz 2008.

37 Vgl. dazu auch Hanfling 2000, S. 96. Es gibt natürlich auch Beispiele von Begriffen, bei deren Bestimmung sich Hanfling wie Jackson eher mit der Frage befassen würde, wieso bestimmte Fälle unter den Begriff fallen, als damit, mit welchem Zweck man den Begriff jeweils verwenden würde. In solchen Fällen würde der o. g. Unterschied zwischen Jacksons und Hanflings Methode wegfallen. Aber gerade in Bezug auf philosophisch interessante Begriffe ist die Frage, wozu die Verwendung dient, häufig interessanter als die Frage, wieso bestimmte Äußerungen, die das betreffende Wort enthalten, wahr sind.

38 Vgl. Jackson 2011, S. 474, Chalmers/Jackson 2001, S. 322.

39 Vgl. Jackson 1998, S. 34, 48. Vgl. auch Jackson 2011, S. 469, Fußnote 3. Dort identifiziert Jackson die Begriffe einer Person mit den Kategorisierungen, die diese Person beherrscht. 
mean by 'knowledge', 'intelligence' or ... and thereby into what their concept of knowledge or intelligence or ... is. ${ }^{40}$

Im betreffenden Artikel macht Jackson allerdings weder deutlich, (a) wieso wir mittels der Methode möglicher Fälle nur zu einer partiellen Antwort auf die Frage gelangen können, wofür wir einen bestimmten Ausdruck gebrauchen, ${ }^{41}$ noch (b) wieso die Methode möglicher Fälle nur teilweise darauf gerichtet ist, zu untersuchen, welches unsere Begriffe sind. ${ }^{42}$ In jedem Fall hängen für Jackson die Extension eines Begriffs und die Bedingungen, unter welchen ein Gegenstand zur Extension eines Begriffs gehört, viel enger mit der Bedeutung des betreffenden Wortes zusammen als bei Hanfling.

\section{Theorien}

Schließlich unterschieden sich Jackson und Hanfling auch darin, wie sie die Verbindung von Begriffen oder der Begriffsanalyse mit Theorien auffassen. Wie wir auf S. 84 gesehen haben, ist Jackson der Ansicht, dass manche unserer Begriffe derart in wissenschaftliche Theorien eingebettet sind, dass sich mit einem wissenschaftlichen Fortschritt erweisen kann, dass wir ein Wort bisher falsch verwendet haben. Außerdem klingt bei Jackson die Idee an, unsere Begriffe würden bestimmte Alltagstheorien implizieren oder manifestieren, wobei diese Alltagstheorien prinzipiell auf der Grundlage empirischer Erkenntnis kritisiert werden können.

Beide Ideen über die Verknüpfung von Theorien und Begriffen werden von Hanfling abgelehnt. Er befasst sich zwar nicht direkt mit Jackson, aber seine Argumentation lässt sich auf Jacksons Ausführungen übertragen. Beginnen wir mit Jacksons Beispiel des Begriffs Fisch. Für die Zeit, in welcher die Leute auch Wale und Delfine zur Extension von Fisch gezählt haben, lassen sich Jackson zufolge die notwendigen und hinreichenden Bedingungen für das Fallen unter diesen Begriff mittels einer Definition der folgenden Art angeben:

40 Jackson 2009, S. 104 (Hervorhebung und Auslassungspunkte von Jackson).

41 Vermutlich schwebt Jackson auch hier wieder vor, dass wir für die Analyse unserer Begriffe nicht nur unsere Klassifikationen von Fällen, sondern auch die theoretische Einbettung dieser Begriffe berücksichtigen müssen. Vgl. S. 83 des vorliegenden Kapitels.

42 Da in den beiden Sätzen im Zitat, wie von mir soeben rekonstruiert, von zwei unterschiedlichen mereologischen Beziehungen die Rede ist, ist mir im Übrigen nicht ganz begreiflich, wieso Jackson den zweiten Satz mit »It follows that « einleitet. Damit diese Einleitung gerechtfertigt ist, muss das »part of« im zweiten Satz sicher im Sinne von »at least part of « verstanden werden, aber auch dann bin ich nicht sicher, ob Jackson mit der Annahme, dass der erste den zweiten Satz impliziert, richtig liegt. Für das hier behandelte Thema ist dies jedoch nicht weiter relevant. 
(6) $\mathrm{x}$ ist genau dann ein Fisch, wenn $\mathrm{x}$ diejenigen Eigenschaften hat, die entsprechend der besten Theorie entscheidend sind für die Zugehörigkeit zu der Art von Wassertieren, die nicht über Beine, aber dafür über Flossen verfügen. ${ }^{43}$

Mit »beste Theorie« meint Jackson nicht die beste zu einem gegebenen Zeitpunkt bekannte Theorie, sondern die Theorie, die sich schließlich und endlich als die beste Theorie herausstellt. ${ }^{44} \mathrm{Im}$ 17. Jahrhundert war demnach die beste Theorie über die entscheidenden Merkmale für die Zugehörigkeit zu den Fischen noch nicht verfügbar. Dennoch haben die Menschen damals mit ihrer Klassifikation von Walen und Delfinen als Fische falsch gelegen, so Jacksons Idee, da sich später herausstellen sollte, dass ein Tier entsprechend der besten Theorie genau dann zu den Fischen gehört, wenn es ein Wirbeltier ist, das ausschließlich oder hauptsächlich über Kiemen atmet. ${ }^{45}$

Gegen diese Auffassung lässt sich mit Hanfling wie folgt argumentieren. ${ }^{46}$ Erstens entspricht (6) nicht der Art und Weise, in welcher das Wort »Fisch« verwendet wird oder im 17. Jahrhundert verwendet wurde. Definitionen wie (6), die auf in Zukunft zu erlangende wissenschaftliche Fortschritte verweisen, mögen, so Hanfling, für theoretische Begriffe wie etwa denjenigen des Gens adäquat sein. ${ }^{47}$ Der Ausdruck»Gen « wurde vom dänischen Botaniker Wilhelm Johannsen für »[d]ieses >etwas in den Gameten bezw. in der Zygote« eingeführt, »welches für den Charakter des Organismus wesentliche Bedeutung hat «. ${ }^{48}$ Die Natur von diesem »etwas« konnte bei der Einführung des Begriffs noch nicht näher bestimmt werden:

Das Wort Gen ist völlig frei von jeder Hypothese; es drückt nur die sichergestellte Tatsache aus, daß jedenfalls viele Eigenschaften des Organismus durch in den Gameten vorkommende besondere, trennbare und somit selbständige »Zustände«, »Grundlagen«, »Anlagen« - kurz, was wir eben Gene nennen wollen - bedingt sind. [...] Damit ist aber noch gar nicht gesagt, daß die Gene selbst chemische Gebilde oder Zustände seien - darüber wissen wir vorläufig noch gar nichts. 49

Bei Jackson (1998, S. 35) eigentlich: $» x$ is a fish iff $x$ has the important properties out of or descended from or explanatory of $F_{1}, F_{2}, F_{3}, \ldots$, according to the best true theory [...] where $F_{1}, F_{2}, F_{3}, \ldots$ are the properties we initially associate with being a fish (the properties of the exemplars) «.

Vgl. Jackson 1998, S. 35: »of course, sometimes we never know what the best theory says; it forever eludes us «.

Vgl. Jackson 1998, S. 34f.

46 Vgl. für eine ähnliche Position auch Carnap 1971 [1950], S. 5 f.

47 Vgl. Hanfling 2000, S. 231.

48 Johannsen 1909, S. 124.

49 Johannsen 1909, S. 124f. 
Der Begriff Fisch ist jedoch ganz anders geartet. Die Existenz von Fischen wurde nicht im Rahmen einer wissenschaftlichen Theorie postuliert, ohne dass klar war, welche Art von Gegenständen Fische überhaupt sind. Wir haben zu Fischen einen direkten Zugang und die meisten von uns begegnen bereits als Kinder konkreten Fisch-Exemplaren. ${ }^{50}$ Und dem Gebrauch des Ausdrucks »Fisch« haftete auch bereits im 17. Jahrhundert nichts Spekulatives an. Es war nicht jede Klassifikation eines Gegenstands als Fisch eine implizite Hypothese oder Vermutung. Dies müsste aber der Fall gewesen sein, wenn der Begriff des Fischs auf eine noch zu etablierende, beste Theorie über die Zugehörigkeit zu den Fischen Bezug genommen hätte. ${ }^{51}$

Diese auf Hanfling basierenden Argumente können wie folgt ergänzt werden: Bestimmten Verwendungen des Ausdrucks »Gen« (z. B. der nichthypothetischen Anwendung auf einen konkreten Gegenstand) musste man sich vernünftigerweise enthalten, solange noch nicht bekannt war, durch welche Komponenten der Geschlechtszellen die Eigenschaften von Individuen (wie z. B. ihre Haarfarbe oder Augenfarbe) festgelegt werden. Eine solche Enthaltung konnte man beim Gebrauch des Ausdrucks »Fisch« nicht beobachten. Zudem könnte man sogar in Frage stellen, ob eine Person, die das Wort »Fisch« im Sinne von (6) versteht, ohne zu wissen, welches die beste Theorie über die Zugehörigkeit zu den Fischen ist, überhaupt über den Begriff Fisch verfügt. Sie kennt unter diesen Voraussetzungen auf jeden Fall keine Methode dafür, Äußerungen der Form »x ist ein Fisch « zu verifizieren, und wäre beispielsweise auch nicht dazu in der Lage, Jacksons Methode möglicher Fälle auf diesen Begriff anzuwenden.

Den im vorangehenden Absatz vorgebrachten Einwänden wäre Jackson nicht ausgesetzt, wenn er (6) so auffassen würde, dass jede Generation das Wort »Fisch« entsprechend der besten zeitgenössischen Theorie verwenden würde. Wenn eine Theorie verworfen wird, wird sie - getreu dem Motto »Die beste Theorie ist tot - lang lebe die beste Theorie « - einfach durch die nächstbeste ersetzt, und so gibt es für jeden Zeitpunkt festgelegte Verifikationsbedingungen

50 Hanfling sagt dies nicht über das Wort Fisch, aber seine Aussagen über andere Begriffe sind auf den vorliegenden Fall übertragbar. Vgl. Hanfling 2000, S. 231.

51 NB: Wenn wir davon ausgehen, dass die im 17. Jahrhundert noch nicht identifizierte, ausschlaggebende Eigenschaft für die Zugehörigkeit zu den Fischen (nämlich eben ein Wirbeltier zu sein, welches hauptsächlich oder ausschließlich über Kiemen atmet) heute Teil des Begriffs Fisch ist, dann hätte zwischen dem 17. Jahrhundert und heute ein Bedeutungswandel stattgefunden, denn (6) wäre mit der aktuellen Definition zwar koextensional, aber nicht synonym. Vgl. Hanfling 2000, S. 229. Dieser Hinweis ist allerdings kein Einwand gegen Jackson, in dessen Ausführungen zu natürlichen Arten im Gegensatz zu denjenigen von Putnam und Kripke - nichts an der Frage hängt, ob ein Bedeutungswandel stattgefunden hat oder nicht. 
für Äußerungen der Art »x ist ein Fisch«, und solche Aussagen können ohne Zurückhaltung getroffen werden. Auch so interpretiert, würden sich für Jackson allerdings Probleme ergeben. Erstens könnte Jackson, so aufgefasst, nicht daran festhalten, dass sich die Leute im 17. Jahrhundert mit ihrer Klassifikation von Walen und Delfinen als Fische geirrt haben. Vorausgesetzt, dass Wale und Delfine entsprechend der besten Theorie des 17. Jahrhunderts zu den Fischen gehörten, lagen die Leute mit dieser Klassifikation richtig. Zweitens müsste sich auch ein begrifflicher Bezug auf die beste zeitgenössische wissenschaftliche Theorie irgendwie in der Art und Weise manifestieren, auf welche der Ausdruck »Fisch « erlernt und das Wort verwendet wird, und eine solche Manifestation fehlt im gewöhnlichen Gebrauch des Ausdrucks.

Inspiriert von der Art und Weise, wie Hilary Putnam und Saul Kripke bisweilen argumentieren, ${ }^{52}$ könnte man gegen diese Diagnose protestieren und darauf bestehen, dass der gewöhnliche Gebrauch des Ausdrucks »Fisch « tatsächlich implizit auf wissenschaftliche Theorien Bezug nimmt. Dies, so könnte man argumentieren, sieht man an der Bereitschaft kompetenter Sprecherinnen und Sprecher, sich von Expertinnen oder Experten über die Extension von Fisch belehren zu lassen. Hätte den Leuten im 17. Jahrhundert ein für sie glaubhaftes Orakel mitgeteilt, die Wissenschaft werde in der Zukunft zum Schluss kommen, dass Delfine und Wale keine Fische sind, so hätten sie diese Klassifikationsweise aller Wahrscheinlichkeit nach adaptiert, was zeigt, dass es mit ihrem Begriff von Anfang an konsistent war, dass sich die gängige, an Oberflächenmerkmalen orientierte Einteilung von Tieren in Fische und Nicht-Fische im Lichte wissenschaftlichen Fortschritts als falsch erweist und dass es für die Zugehörigkeit zu den Fischen ausschlaggebende Merkmale gibt, die bisher nicht bekannt sind. Ihr Begriff muss also in der Art von (6) definiert werden, und eine Definition wie (7) ist nicht adäquat:

(7) $\mathrm{x}$ ist genau dann ein Fisch, wenn $\mathrm{x}$ im Wasser lebt und keine Beine, aber Flossen hat.

Dies ist jedoch ein Non sequitur. Das geschilderte Szenario impliziert nicht, dass der etablierte Fischbegriff einen Bezug auf eine wissenschaftliche Theorie enthält, sondern lässt sich auch ganz einfach (und plausibler) damit erklären, dass die Leute bereit sind, ihren Fischbegriff im Lichte wissenschaftlicher Erkenntnisse zu revidieren.

Hanfling bestreitet nicht, dass es angesichts von wissenschaftlichen Fortschritten hilfreich sein kann, neue Begriffe einzuführen oder alte Begriffe zu verändern. ${ }^{53}$ Und er räumt sogar ein, dass beispielsweise der heute etablierte

52 Vgl. z. B. Putnam 1975, S. 237f., besprochen in Hanfling 2000, S. $228 f$.

53 Vgl. Hanfling 2000, S. 236. 
Begriff Gold einen Bezug auf ExpertInnen impliziert beziehungsweise darauf, was ExpertInnen (nämlich entweder Juwelierinnen und Juweliere oder NaturwissenschaftlerInnen) auf der Grundlage von Methoden, die dem Laien nicht bekannt sind, als Gold deklarieren würden. ${ }^{54}$ Aber seiner Auffassung nach kann die Bedeutung eines Ausdrucks nicht in der Weise von (6) auf eine ideale wissenschaftliche Theorie Bezug nehmen. Eine Auffassung der Bedeutung eines sprachlichen Ausdrucks, nach welcher wir nie sicher sein können, ob wir den Ausdruck nun richtig oder falsch verwenden (da sich jederzeit herausstellen könnte, dass die gegenwärtig verfügbare Theorie, nicht die beste Theorie ist), ist Hanfling zufolge inadäquat. ${ }^{55}$

Kommen wir nun zur zweiten Art und Weise, in welcher Begriffe Jackson zufolge mit Theorien verknüpft sind. Jackson zufolge manifestiert sich im gängigen Gebrauch der Ausdrücke »freiwillig «, »wissen« und »Überzeugung« die Alltagstheorie darüber, was freier Wille, Wissen und Überzeugungen sind. ${ }^{56}$ Hanfling setzt sich mit dieser Idee im Zusammenhang mit Patricia und Paul Churchlands Eliminativismus auseinander. Dieser Position zufolge stellt sich unsere Alltagspsychologie (»folk psychology«) im Zuge des neurowissenschaftlichen Fortschritt als falsch heraus, und deshalb müssen unsere mentalen Begriffe (wie etwa Wunsch, Überzeugung oder Schmerz), die diese Alltagstheorie implizieren, durch adäquatere Begriffe ersetzt werden. ${ }^{57}$ Hanfling hält dieser Auffassung entgegen, dass erstens die Sätze, von denen behauptet wird, dass sie zur Alltagstheorie gehören, keine Hypothesen sind, die sich widerlegen lassen, dass zweitens unsere mentalen Begriffe ihre Funktion (z. B. im Zusammenhang mit der Erklärung menschlichen Verhaltens) perfekt erfüllen und deshalb nicht einsichtig ist, wie sie sich als inadäquat herausstellen könnten, ${ }^{58}$ und dass drittens die Begriffe aus den Neurowissenschaften ganz anders funktionieren als unsere mentalen Begriffe und sie überhaupt nicht dafür geeignet wären, Letztere zu ersetzen bzw. abzulösen. ${ }^{59}$ Ich werde nachfolgend nur auf den ersten von Hanfling vorgebrachten Punkt näher eingehen, da dieser für das vorliegende Kapitel die größte Relevanz hat.

Teil der Alltagspsychologie soll zum Beispiel sein:

\footnotetext{
54 Vgl. Hanfling 2000, S. 229.

55 Vgl. Hanfling 2000, S. $238 f$.

56 Vgl. Jackson 1998, S. 31 .

57 Vgl. Hanfling 2000, S. 245f., 250. Vgl. für die gesamte nachfolgende Diskussion zur Alltagspsychologie Hanfling 200o, Kap. 13 und für eine damit zusammenhängende Thematik auch Hanfling 2000, Kap. 11.

$5^{8}$ Vgl. Hanfling 2000, S. 249f., 255.

59 Vgl. Hanfling 200o, insb. S. 255, $258 \mathrm{f}$.
} 
(i) dass Personen, die den Wunsch haben, dass $p$, und die Überzeugung, dass ein bestimmtes Verhalten $Q$ dazu führen würde, dass $p$, $Q$ ausführen werden, sofern sie die Möglichkeit dazu haben und keine anderen Wünsche und Überzeugungen haben, die gegen $Q$ sprechen,

(ii) dass jemand, der befürchtet, dass $p$, sich wünscht, dass nicht-p, und

(iii) dass jemand, der aufrichtig behauptet, dass $p$, die Überzeugung hat, dass $p .{ }^{60}$

Diese Sätze sind jedoch analytisch und somit nicht Teil einer Theorie. ${ }^{61}$ Es kann sich nicht herausstellen, dass es den beschriebenen Zusammenhang zwischen Befürchtungen und Wünschen nicht gibt, weil dieser Zusammenhang sich aus den Bedeutungen von »befürchten« und »sich wünschen« ergibt. $^{62} \mathrm{Im}$ Übrigen ist es contra Patricia und Paul Churchland auch nicht möglich, dass sich herausstellt, dass es in Wirklichkeit keine Wünsche, Befürchtungen oder Überzeugungen gibt: Wenn wir über jemanden sagen, er habe eine bestimmte Überzeugung, dann entspricht dies keinem Postulat: Die Behauptung, dass mein Freund den Wunsch hat, schwimmen zu gehen, ist keine Behauptung über einen versteckten Vorgang oder eine erforschbare Entität, der resp. die sich in seinem Gehirn abspielt resp. befindet.63 Das Urteil, dass es Personen gibt, die über intentionale Zustände verfügen, ist zwar kontingent, aber dass es wahr ist, ist nicht weniger offensichtlich, als dass es Menschen gibt, und kann sich deshalb auch nicht im Zuge des wissenschaftlichen Fortschritts als falsch erweisen. ${ }^{64}$

Wie sich gezeigt hat, weicht Jacksons Methode möglicher Fälle in verschiedener Hinsicht von der Methode typischer Fälle ab, wie sie von Wittgenstein empfohlen und unter anderem von Hanfling angewendet wird. Die bisher im vorliegenden Kapitel besprochenen Kritikpunkte an Jacksons Methode lassen aber offen, dass diese Methode zur Analyse philosophischer Begriffe (unter Absehung beispielsweise von der Rolle, die wissenschaftliche Theorien darin spielen) für die Ordinary Language-Philosophie hilfreich sein

6o Vgl. Hanfling 2000, S. 251, 254.

61 Im Falle von bestimmten Urteilen, die zur Alltagspsychologie gezählt werden, lässt sich bestreiten, dass sie analytisch sind, aber in Bezug auf diese Urteile muss dann folgerichtig auch gesagt werden, dass sie nicht aus unseren mentalen Begriffen hervorgehen und dass ihre Erweisung als falsch deshalb nicht zeigen kann, dass unser Begriffssystem inadäquat ist.

62 Vgl. Hanfling 2000, S. 251f., 26of.

63 Vgl. Hanfling 2000, S. 254.

64 Vgl. für einen ähnlichen Punkt Hanfling 2000, S. 251. Dass es in Wirklichkeit keine Wünsche, Überzeugungen usw. gibt, wird von Paul und Patricia Churchland explizit als plausible Hypothese erachtet: Vgl. z. B. Churchland 1986, S. 397, und Churchland 1991, S. 65, besprochen bei Hanfling 2000, S. 254 und 260. 
kann. Nachfolgend wende ich mich einigen Einwänden gegen die Nützlichkeit der Methode möglicher Fälle zu.

\section{$3 \quad$ Einwände}

Die kritische Literatur, die in den letzten Jahren zur Methode möglicher Fälle publiziert worden ist, problematisiert vor allem den Stellenwert, den die Verfechter der Methode Intuitionen zuschreiben. Entweder wird die Aussagekraft davon hinterfragt, welche Intuitionen einzelne Philosophinnen und Philosophen haben, oder ganz allgemein dafür argumentiert, dass Intuitionen kein argumentativer Wert zukommt. Beide Bedenken werden mitunter vonseiten der Experimentellen Philosophie vorgebracht. ${ }^{65}$ Auf die Frage, ob die Ergebnisse der Begriffsanalyse aussagekräftiger wären, wenn sie in großangelegten Umfragen erhoben und nicht nur auf den Intuitionen (oder auf dem Sprachgefühl) einzelner Philosophinnen und Philosophen beruhen würden, werde ich an anderer Stelle eingehen. ${ }^{66}$ Aber auch die Frage, ob Philosophinnen und Philosophen sich im Allgemeinen für (eigene oder fremde) Intuitionen interessieren sollten (was auch immer mit »Intuition « gemeint ist), soll hier nicht beantwortet werden: Wie bereits weiter oben erwähnt, legt Jackson, um dessen Auffassung der Methode möglicher Fälle es hier geht, auf den Einbezug des Begriffs der Intuition bei der Beschreibung seines Vorgehens keinen Wert. Wenn wir jemanden fragen, ob er die Intuition habe, dass ein Akteur in einem Gettier-Fall über Wissen verfüge, so Jackson, wollen wir nichts anderes wissen, als ob die Person den Akteur so beurteilt, dass er über Wissen verfügt. ${ }^{67}$

Der interessanteste Artikel, der übrig bleibt, wenn wir von der kritischen Literatur zur Methode möglicher Fälle absehen, in denen es um die Aussagekraft von Intuitionen geht, ist »Empirical Metaphysics: The Role of Intuitions about PossibleCases in Philosophy «(2008) von Janice Dowell. In Dowells Artikel wird nicht die Brauchbarkeit der Methode möglicher Fälle in Frage gestellt, sondern Jacksons (und Chalmers) Auffassung dieser Methode kritisiert. ${ }^{68}$ Die nachfolgende Besprechung der von Dowell aufgeworfenen Fragen dient einerseits dazu, einige Aspekte von Jacksons Konzeption der Methode möglicher Fälle noch einmal deutlicher herauszuarbeiten. Andererseits soll im Rahmen

65 Vgl. z. B. Alexander/Weinberg 2007, S. 6of. Die Bedenken werden aber auch außerhalb der experimentellen Philosophie vorgebracht. Vgl. z. B. Nagel 2012 und Mizrahi 2014.

66 Vgl. insb. das Kapitel 7 des vorliegenden Buchs.

67 Vgl. Jackson 2011, S. 471. Ich komme auf das Thema Intuitionen im Kapitel 6 der vorliegenden Abhandlung zurück.

68 Von Chalmers' Beteiligung werde ich in der nachfolgenden Diskussion absehen. 
der Diskussion auch auf einen zentralen Aspekt von Hanflings Methode und der in der vorliegenden Abhandlung verteidigten Philosophie-Konzeption hingewiesen werden.

Dowells Argument hängt mit Jacksons Idee zusammen, dass unser Wissen darüber, ob ein bestimmter möglicher Fall in die Extension eines Begriffs fällt, den wir besitzen, in einem bestimmten Sinn a priori ist. ${ }^{69}$ Jacksons Auffassung ist die folgende: Bei der Begriffsanalyse handelt es sich generell um ein empirisches Unterfangen, und zwar auch dann, wenn eine Philosophin ihre eigenen Begriffe analysiert und sich nicht dafür interessiert, in welchem Umfang sich ihre Begriffe mit den in ihrer Sprachgemeinschaft etablierten Begriffen decken. Es ist deshalb ein empirisches Unterfangen, so Jackson, weil ein wesentlicher Bestandteil der Analyse - beispielsweise ihres Wissensbegriffs darin besteht, dass die Philosophin sich darüber klar wird, welche Art von Fällen sie als Fälle von Wissen klassifiziert. Und dass sie diese Fälle so klassifiziert, ist eine empirische Tatsache. ${ }^{70}$ Wenn wir unseren eigenen Wissensbegriff analysieren und dabei mögliche Fälle in Fälle von Wissen und Fälle von Nicht-Wissen einteilen, ist dieses Unterfangen aber immerhin insofern a priori, so Jackson weiter, als wir, um zu beurteilen, ob ein Fall ein Fall von Wissen ist oder nicht, nicht wissen müssen, ob es sich bei diesem Fall um einen echten oder einen erfundenen Fall handelt. Unsere Beantwortung der Frage, ob ein bestimmter Fall ein Fall von Wissen ist, hängt also nicht davon ab, welche Welt wir für die aktuale Welt halten. ${ }^{71}$

Dowell zufolge ist der zuletzt dargelegte Teil von Jacksons Argumentation nicht stichhaltig. Bei Jackson lesen wir in diesem Zusammenhang zum Beispiel:

When we do conceptual analysis of $K$-hood, we address the question of what it takes to be a $K$ in the sense of when it is right, and when it is wrong, to describe some situation in terms of ' $K$ ', and so we make explicit what our subject is when we discuss $K$ s. The part of this enterprise that addresses the question of what things are $K$ at a world, under the supposition that that world is the actual world, is the a priori part of conceptual analysis, because the answer depends not at all on which world is in fact the actual world $[\ldots] .{ }^{72}$

Um den Begriff $K$ zu analysieren, müssen wir uns Jackson zufolge überlegen, welche Gegenstände $K$ sind in einer bestimmten Welt $w_{p}$, unter der Annahme,

69 Ich weiche hier relativ stark von Dowells Formulierungen ab, damit ihr Einwand besser zum Wortlaut der oben rekonstruierten Texte von Jackson passt. Der springende Punkt ihrer Argumentation bleibt dabei bestehen.

$70 \quad$ Vgl. Jackson 1998, S. 47.

71 Vgl. Jackson 1998, S. 51.

72 Jackson 1998, S. 51. 
diese Welt sei die aktuale Welt $w_{a}$. Gehen wir davon aus, dass wir es mit einem Fall zu tun haben, in dem $w_{1}$ von $w_{a}$ verschieden ist - oder besser: in dem wir glauben, dass $w_{1}$ von $w_{a}$ verschieden ist. Dowell versteht Jackson so, dass wir uns, um festzustellen, wie wir den Ausdruck » $K$ « tatsächlich verwenden, in einem solchen Fall so gut es geht in eine Situation hineinfühlen müssen, in der $w_{7}$ die aktuale Welt ist, um dann die Gegenstände in $w_{7}$ entsprechend dieser Simulation in Ks und Nicht-Ks einzuteilen. Der springende Punkt von Dowells Argumentation ist dann: Was wir über die Verteilung von $K \mathrm{~s}$ in $w_{l}$ sagen, wenn wir uns bloß vorstellen, $w_{\imath}$ wäre $w_{a}$, ist hinsichtlich unserer tatsächlichen Sprachverwendung weniger aussagekräftig als das, was wir über die Verteilung von $K$ s in $w_{1}$ sagen, wenn wir tatsächlich glauben, dass $w_{1}$ mit $w_{a}$ identisch ist.

Dowell geht davon aus, dass Jacksons Begriffsanalyse eine Erforschung unserer Dispositionen zur Verwendung sprachlicher Ausdrücke ist. Wenn wir beispielsweise im Rahmen eines epistemologischen Gedankenexperiments mit einem fiktionalen Szenario konfrontiert werden und beurteilen sollen, ob dieses Szenario einen Fall von Wissen darstellt oder nicht, dann soll Folgendes herausgefunden werden: Sind wir dazu disponiert, in Fällen wie dem beschriebenen Szenario zu sagen, dass der relevante Akteur über Wissen verfügt? ${ }^{73}$ Um diese Frage zu beantworten, »stellen wir uns vor, zu glauben«, dass das beschriebene Szenario ein echter Fall ist - das Szenario also für wahr zu halten - und beobachten, wie wir sprachlich darauf reagieren. Das Problem ist nun, so Dowell, dass Szenarien, von denen wir uns nur so gut es geht vorstellen, sie seien aktual, unsere Dispositionen nicht so zuverlässig auslösen wie Szenarien, von denen wir tatsächlich glauben, dass sie aktual sind. Wenn wir sagen, dass wir in einem bestimmten Gettier-Fall kein Wissen zuschreiben würden, aber dann in einen identisch strukturierten Fall geraten und sagen, es sei ein Fall von Wissen, haben wir unser Lehnstuhl-Urteil über unsere Sprachverwendung falsifiziert. Und insofern ist die Begriffsanalyse Dowell zufolge auch in Jacksons Sinn a posteriori: Um eine Behauptung darüber, was wir über ein bestimmtes Szenario sagen würden, zu verifizieren, müssen wir tatsächlich glauben, dass dieses Szenario aktual ist. Und somit hängen unsere Urteile darüber, wie wir sprachliche Ausdrücke verwenden, von unseren Überzeugungen davon ab, welche Welt aktual ist.

73 Besser wäre es, in solchen Zusammenhängen von Dispositionen dazu zu sprechen, bestimmte Fragen mit »Ja« oder »Nein« zu beantworten bzw. bestimmten Klassifikationen zuzustimmen (vgl. das, was Quine, z. B. in Word and Object, unter dem Label »dispositions to assent or dissent to a sentence « beschreibt). Denn natürlich sind wir in den meisten Fällen, in denen wir beispielsweise annehmen, jemand verfüge über Wissen in Bezug auf $p$, nicht dazu disponiert, diese Annahmen unaufgefordert zu verbalisieren. Diese Problematik werde ich nachfolgend ignorieren. 
Um ihren Punkt deutlicher zu machen, führt Dowell einen Fall an, in welchem es nicht um sprachliche Dispositionen, sondern um Mut geht. Dowell fasst Mut als die Disposition auf, sich Gefahren auszusetzen, wenn dies für die Erreichung eines wertvollen Ziels erforderlich ist, und stellt die Frage, in welchem Maß wir aus dem Lehnstuhl beurteilen können, ob wir mutig sind oder nicht. Offensichtlich kann meine Einschätzung davon, ob ich mich in einer bestimmten Situation mutig verhalten würde oder nicht, falsifiziert werden dadurch, dass ich in die betreffende Situation gerate und mich anders als erwartet verhalte. Und zwar unabhängig davon, wie realistisch und detailgetreu mir die vorgestellte Situation beschrieben wird und wie sehr ich mich anstrenge, mich in die Situation hineinzuversetzen. Und wie mutig ich bin, hängt davon ab, wie ich mich tatsächlich verhalte, und nicht von meiner Einschätzung davon, wie ich mich in kontrafaktischen Situationen verhalten würde. ${ }^{74}$ Wenn Dowell Recht damit hat, dass der Mut-Fall in der entscheidenden Hinsicht gleich geartet ist wie der Fall, in welchem wir versuchen, unsere Verwendung eines sprachlichen Ausdrucks zu klären, dann wäre ihr Argument gegen Jackson sicher stichhaltig.

Tatsächlich räumt Dowell ein, dass die beiden Fälle - je nachdem, mit welchen konkreten Ausdrücken wir es auf der Seite der sprachlichen Dispositionen zu tun haben - nicht ganz analog funktionieren. Dies deshalb, weil jemandes Verhalten in einer gefährlichen Situation stark von der Angst beeinflusst wird, die ihn in der Situation befällt, und diese Angst aus dem Lehnstuhl nur bedingt heraufbeschwört werden kann. Diese emotionale Komponente fehlt beispielsweise bei der Verwendung des Ausdrucks »wissen «. In anderen Fällen, zum Beispiel bei der Verwendung der Ausdrücke »abstoßend« oder »niedlich«, mag es, so Dowell, anders aussehen, weshalb diese Fälle sprachlicher Dispositionen dem Mut-Fall ähnlicher wären als der Fall des Gebrauchs des Wortes »wissen $\ll .75$

Wie bereits weiter oben erwähnt, ist es nicht Dowells Absicht, zu zeigen, dass die Methode möglicher Fälle zur Analyse eines Begriffs vollkommen unbrauchbar ist. Ihr zufolge sind die für die Analyse von Begriffen relevanten Urteile über die Art und Weise, wie wir sprachliche Ausdrücke verwenden, nicht a priori und können prinzipiell durch Erfahrung falsifiziert werden. Das heißt aber nicht, dass das, was wir aus dem Lehnstuhl darüber sagen, wie wir einen bestimmten, konstruierten Fall beurteilen würden, kein guter Hinweis darauf ist, wie wir die fraglichen sprachlichen Ausdrücke tatsächlich verwenden. Wenn wir beispielsweise wissen wollen, ob wir Gettier-Fälle als

74 Vgl. Dowell 2008, S. 29f.

75 Vgl. Dowell 2008, S. 30. 
Fälle von Wissen bezeichnen würden, und nicht die Möglichkeit haben, einen echten Gettier-Fall zu erschaffen, dann sind Gedankenexperimente unsere beste Grundlage zur Erlangung von Hypothesen über unsere Dispositionen zur Verwendung von »wissen « in den relevanten Kontexten. Auch Dowell fasst die Methode möglicher Fälle deshalb als ein nützliches Instrument bei der Reflexion darüber auf, was unsere sprachlichen Ausdrücke bedeuten. ${ }^{76}$

Mit Blick auf Jacksons Ausführungen dazu, wie Begriffe analysiert werden und inwiefern es sich bei der Begriffsanalyse um ein A priori-Unterfangen handelt, lässt sich Dowells Argument einiges entgegenhalten. Zunächst einmal ist es alles andere als offensichtlich, dass es Jackson um eine Erforschung unserer Dispositionen zur Verwendung sprachlicher Ausdrücke geht. In den Texten, in denen sich Jackson sich mit der Begriffsanalyse befasst, kommen die Wörter »disposition« oder »disposed « äußerst selten und nur zweimal im Zusammenhang mit unserer Verwendung sprachlicher Ausdrücke vor. ${ }^{77}$ Wie bereits weiter oben erwähnt, betont Jackson außerdem, dass er sich nicht für unsere spontanen Reaktionen auf Fälle interessiert, mit denen wir konfrontiert werden, sondern für bedachte Urteile darüber, ob ein Fall unter einen bestimmten Begriff fällt, die auch einer Diskussion standhalten:

\begin{abstract}
A person's first-up response as to whether something counts as a $K$ may well need to be discounted. One or more of: the theoretical role they give $K$-hood, evidence concerning other cases they count as instances of $K$, signs of confused thinking on their part, cases where their classification is, on examination, a derivative one (they say it's a $K$ because it is very obviously a $J$, and they think, defeasibly, that any $J$ is a $K$ ), their readiness to back off under questioning, and the like, can justify rejecting a subject's first-up classifications as revealing their concept of $K$-hood. ${ }^{78}$
\end{abstract}

Gegenüber den Stellen, die nahelegen könnten, dass Jackson herausfinden will, welche sprachlichen Ausdrücke wir tatsächlich in welchen Situationen äußern, ${ }^{79}$ gibt es im Übrigen viel mehr Stellen, an denen zum Ausdruck kommt, dass Jackson sich für normative Urteile interessiert: Es geht ihm um »intuitions

76 Vgl. Dowell 2008, S. 45 .

77 Jackson 1998, S. 2, Jackson 2010, S. 181. In Jackson 2009 und 2011 ist an keiner Stelle von Dispositionen die Rede, und in Chalmers/Jackson 2001 nicht im Zusammenhang mit unserem Sprachgebrauch. In die Nähe einer Disposition kommt in Jackson 2011 immerhin die »inclination to say [something] « (S. 477; vgl. für eine ähnliche Ausdrucksweise auch Chalmers/Jackson 2001, S. 346) und in Jackson 1998 das »Triggern« von Äußerungen (S.65), aber selbst diese Formulierungen kommen kein weiteres Mal in Jacksons Publikationen zur Begriffsanalyse vor.

78 Jackson 1998, S. 35. Vgl. auch Jackson 2010, S. 173.

79 Vgl. Jackson 1998, S. 47, Jackson 2010, S. 184. 
about how various cases $[. .$.$] are correctly described { }^{80}$ und die Analyse des Begriffs $K$ erfolgt ihm zufolge auf der Grundlage der Frage, »when it is right, and when it is wrong, to describe some situation in terms of $>K_{4} \ll .{ }^{81}$ Bezugnehmend auf diesen Umstand lässt sich zwar kein Knock-down-Argument gegen die Auffassung konstruieren, dass der Gegenstand von Jacksons Untersuchung Dispositionen sind. Es könnte ihm um Dispositionen zu wohlüberlegten, normativen Urteilen gehen, und um Dispositionen dazu, in Diskussionen an Urteilen festzuhalten. Aber bei unseren Dispositionen zu wohlüberlegten, normativen, stabilen Urteilen scheint es mir weniger einsichtig zu sein als bei unseren Dispositionen zum spontanen Sprachgebrauch, weshalb sie von konstruierten Fällen im Gegensatz zu echten Fällen, denen wir im Alltag begegnen, nur unzuverlässig ausgelöst werden sollten. Dies unter anderem deshalb, weil in die relevanten Reflexionen und Diskussionen, wie auch Jackson im obigen Zitat anmerkt, typischerweise ohnehin auch andere, konstruierte Fälle einfließen. Um uns darauf festzulegen, wie ein bestimmter Fall beschrieben werden sollte, überlegen wir uns, wie wir andere, ähnlich gelagerte oder in spezifischen Hinsichten abweichende Fälle beschreiben würden. Ob der zentrale, ursprünglich zu beurteilende Fall echt oder konstruiert ist, scheint im Lichte dieses Umstands nicht besonders relevant zu sein.

Und wenn es Jackson nicht um deskriptive, sondern um normative Urteile geht, können wir sogar auf Dowells eigene Argumentation verweisen, um gegen die Relevanz des Unterschieds zwischen echten und konstruierten Fällen zu argumentieren. Dowell deutet nämlich in der Passage zur Disposition, sich mutig zu verhalten, an, dass wir zwar aus dem Lehnstuhl beurteilen können, wie wir uns in einer gefährlichen Situation verhalten sollten, aber nicht, wie wir uns verhalten würden. ${ }^{82}$ Die Fragen, die Jackson im Rahmen der Analyse von Begriffen stellt, setzt Dowell in Analogie zur Frage, wie wir uns in einer gefährlichen Situation verhalten würden. Wenn Jackson sich aber in Wirklichkeit für unsere Dispositionen dazu interessiert, Beschreibungen von Fällen als korrekt zu erachten, bezieht sich seine Frage eher auf unsere Urteile darüber, wie wir uns verhalten sollten, als wie wir uns verhalten würden, und Dowell müsste mithin einräumen, dass wir keine echten Fälle benötigen, um die entsprechenden Dispositionen auszulösen.

Prima facie könnte man auf die Idee kommen, Dowell ganz grundsätzlich mangelndes Wohlwollen bei der Interpretation von Jacksons Ausführungen vorzuwerfen:Würde es Jackson um die Erforschung von Verhaltensdispositionen

8o Jackson 1998, S. 31. Hervorhebung von mir. Vgl. auch Jackson 1998, S. 37.

81 Jackson 1998, S. 51.

82 Vgl. Dowell 2008, S. 29. 
gehen, so könnte man sagen, wäre es geradezu absurd, dass er die Begriffsanalyse als $A$ priori-Unterfangen einstuft. Denn offensichtlich sind Urteile darüber, welche Verhaltensdispositionen wir haben, a posteriori. Diese Überlegung beruht jedoch auf einem Missverständnis. Denn Jackson verwendet »a priori« in einem idiosynkratischen Sinn:

(8) Ein Urteil ist genau dann a priori, wenn wir seinen Wahrheitswert kennen können, ohne zu wissen, welche Welt aktual ist. ${ }^{83}$

Nun könnte man natürlich sagen: Dass ich Wörter auf die Weise verwende, in der ich sie verwende, ist kontingent. Es gibt eine mögliche Welt, in der ich »Hammer « zur Bezeichnung von Schraubenziehern verwende. Um zu wissen, wie ich das Wort »Hammer verwende, muss ich also wissen, welches die aktuale Welt ist. Diese Überlegung entspricht aber nicht Jacksons Idee, die sich wie folgt rekonstruieren lässt: Um zu wissen, dass ich ein Werkzeug, das dafür gemacht ist, Nägel in die Wand zu schlagen, als Hammer bezeichnen würde, benötige ich nicht die geringste Ahnung darüber, in welcher Welt ich mich befinde und ob es in dieser Welt überhaupt Werkzeuge gibt. Und in diesem Sinn ist dieses Urteil gemäß (8) a priori. Natürlich ist es dennoch eine kontingente Tatsache, dass ich das Wort »Hammer« so verwende, wie ich es verwende. Und Jackson zufolge ist ja auch die Analyse meiner eigenen Begriffe ein empirisches Unterfangen. Nur in einem sehr spezifischen, idiosynkratischen Sinn ist es ihm zufolge eben a priori. Und diese idiosynkratische Auffassung der Apriorität verwendet er dazu, einen bestimmten Unterschied hervorzuheben zwischen Begriffen wie Hammer, deren Extension in kontrafaktischen Welten nicht davon abhängt, was in der aktualen Welt der Fall ist, und so genannt »zweidimensionalen « Begriffen wie Wasser. Die Extension des Begriffs Wasser in einer kontrafaktischen Welt hängt davon ab, welche Mikrostruktur Wasser in der aktualen Welt hat. Ohne über Wissen darüber zu verfügen, welche Welt aktual ist, kann ich Jackson und anderen Vertreterinnen und Vertretern des Zweidimensionalismus zufolge die Frage, ob ich eine grüne, schleimige, hochgiftige Substanz mit der Mikrostruktur $\mathrm{H}_{2} \mathrm{O}$ als Wasser bezeichnen würde, nicht beantworten. Ich müsste zunächst wissen, welche Mikrostruktur die durchsichtige, trinkbare Substanz, die vom Himmel regnet und die Meere füllt, ${ }^{84}$ in der aktualen Welt hat (sofern es in der aktualen Welt eine solche Substanz gibt). Unabhängig davon, ob wir dieser Auffassung von Jackson zustimmen wollen oder nicht, ist sein Urteil, die Begriffsanalyse sei a priori, wenn es so verstanden wird wie im vorliegenden Absatz ausgeführt, damit vereinbar,

83 Vgl. Jackson 1998, S. 51.

84 Die Formulierung ist von der Wasser-Definition von Nimtz inspiriert. Vgl. Nimtz 2012, S. 235 . 
dass sie im gängigen Sinn empirisch ist, und deshalb ist Dowells Idee, es gehe Jackson um die Erforschung von Verhaltensdispositionen, nicht von vornherein absurd.

Ich habe bisher Dowells Argument so dargestellt, dass es auf alle Fragen der Art »Wie würdest du eine Situation beschreiben, die soundso geartet ist?«, »Würdest du sagen, ein Objekt, das soundso geartet ist, sei ein F? « usw. Anwendung findet. Einige Passagen ihres Artikels legen jedoch nahe, dass sich Dowells Einwand gegen Jackson nur auf eine ganz bestimmte Formulierung solcher Fragen bezieht, nämlich auf die folgende:

(9) Wie würdest du eine Situation beschreiben, die soundso geartet ist angenommen, sie trete in der aktualen Welt ein?

Eine ähnliche Formulierung kam bereits im Zitat auf S. 97 des vorliegenden Kapitels vor. Ich möchte nun die Gründe dafür ausführen, dass sich Vertreterinnen und Vertreter zweidimensionaler Bedeutungstheorien zur Verwendung solcher Formulierungen veranlasst sehen. Zu diesem Zweck muss ich noch einmal detaillierter auf die Begrifflichkeit des Zweidimensionalismus eingehen: Der Zweidimensionalismus unterscheidet zwischen $A$-Extensionen und $C$-Extensionen von Ausdrücken sowie zwischen $A$-Intensionen und $C$-Intensionen. Die $A$-Extension eines Ausdrucks in einer Welt $w$ ist die Extension, die dieser Ausdruck in $w$ unter der Annahme hat, dass $w$ die aktuale Welt ist. Die C-Extension in $w$ ist die Extension, die ein Ausdruck in $w$ unter der Annahme hat, dass unsere tatsächliche Welt die aktuale Welt ist (»C $C$ «steht für »counterfactual«). Die A-Intension eines Ausdrucks $a$ ist die Funktion, die Welten auf $A$-Extensionen von $a$ abbildet, die $C$-Intension die Funktion, die Welten auf $C$-Extensionen abbildet. ${ }^{85}$ Jackson zufolge besitzen wir im oben spezifizierten Sinn A priori-Wissen über die $A$-Intensionen unserer Ausdrücke und somit auch über die $C$-Intensionen von Ausdrücken, deren $A$-Intensionen sich nicht von ihren $C$-Intensionen unterscheiden (wie z. B. »Hammer«). Bei Ausdrücken, deren Extension in einer Welt $w$ davon abhängt, ob wir $w$ als aktual oder als kontrafaktisch betrachten (wie z. B. »Wasser«), ist uns die $A$-Intension, aber nicht die $C$-Intension a priori zugänglich. ${ }^{86}$

Vor dem Hintergrund dieser begrifflichen Spezifikationen wird einsichtig, weshalb Zweidimensionalistinnen und Zweidimensionalisten Formulierungen wie (9) verwenden. Wir können im Rahmen der Beurteilung von Gedankenexperimenten nicht einfach fragen: »Wie würdest du eine Situation beschreiben, die soundso geartet ist?«, sondern müssen entweder fragen:

$85 \quad$ Vgl. Jackson 1998, S. 48.

86 Vgl. Jackson 1998, S. 49, 51f. 
(10) Wie würdest du eine Situation beschreiben, die soundso geartet ist angenommen, sie trete in der aktualen Welt ein?

Oder:

(11) Wie würdest du eine Situation beschreiben, die soundso geartet ist angenommen, unsere tatsächliche Welt sei die aktuale Welt?

Die Frage (10) zielt dabei auf die Identifikation der $A$-Intensionen sprachlicher Ausdrücke ab und ihre Beantwortung ist mithin nach Jackson im Gegensatz zu (11) im oben spezifizierten Sinn a priori. In ihrer Kritik an der These, dass solche Urteile a priori sind, fasst Dowell die Formulierung »angenommen, sie trete in der aktualen Welt ein« so auf, dass wir uns, um Fragen des Typus (10) zu beantworten, in eine Welt hineinversetzen müssen, in der die betreffende Situation eingetreten ist, und das bedeutet nach Dowell, dass wir uns so gut es geht in eine Person einfühlen müssen, die tatsächlich glaubt, dass diese Situation eingetreten ist. ${ }^{87}$ Die Idee dabei ist, dass die resultierenden, »simulierten Überzeugungen unsere Sprachgebrauchs-Dispositionen auslösen sollen, die normalerweise von echten Überzeugungen ausgelöst werden. Und Dowells Argument gegen Jackson stellt, wie bereits oben erwähnt, darauf ab, dass vorgegebene Überzeugungen unsere Dispositionen nicht so zuverlässig auslösen wie echte Überzeugungen, und dass die Sprachgebrauchs-Urteile, die wir in Bezug auf hypothetische Situationen fällen, deshalb von unserem Sprachgebrauch in Fällen widerlegt werden können, in denen wir tatsächlich davon überzeugt sind, dass die betreffenden Situationen eingetreten sind.

Mir erscheint es allerdings alles andere als selbstverständlich, dass wir uns, um hypothetische Urteile der für Jackson relevanten Art zu fällen, in die betreffenden Situationen hineinversetzen müssen, und zwar unabhängig davon, ob die betreffenden Fragen eine »unter der Annahme, ...«-Ergänzung umfassen oder nicht. Wenn man mich fragt: »Würdest du einen 25-jährigen Mann, der unverheiratet und kein Geistlicher oder Ähnliches ist, als Junggesellen bezeichnen?«, dann muss ich mir nicht vorstellen, dass ein Mann mit diesen Eigenschaften tatsächlich existiert, geschweige denn dass so ein Mann in Fleisch und Blut vor mir steht, um zu wissen, dass die gestellte Frage mit »Ja« zu beantworten ist. Und daran ändert sich auch nichts, wenn wir die Frage wie oben erwähnt ergänzen: »..., wenn wir davon ausgehen, dass dieser Mann Teil der aktualen Welt ist«. Worauf ich mich zur Beantwortung der Frage, ob ich einen 25-jährigen, unverheirateten Mann, der kein Geistlicher oder

87 Bei Dowell ist in diesem Kontext von »pretend-belief« die Rede und davon, Überzeugungen zu imitieren (»mimic«). Vgl. Dowell 2008, S. 28. Bei dieser Interpretation von »supposition« beruft sie sich auf Robert Stalnaker (1987, S. 79-81). Vgl. Dowell 2008, S. 28, Fußnote 25. 
Ähnliches ist - wenn dieser Mann Teil der aktualen Welt ist -, als Junggesellen bezeichnen würde, berufe, ist ganz einfach die sprachliche Regel, dass »Junggeselle« auf alle unverheirateten Männer im heiratsfähigen Alter Anwendung findet, die keine Geistlichen oder Ähnliches sind. Und selbst wenn wir den Sachverhalt unter Rückgriff auf das Auslösen von Dispositionen beschreiben wollen, sehe ich nicht, weshalb wir echte Situationen (bzw. solche, die wir für echt halten) benötigen, um Urteile über unsere Dispositionen zu verifizieren oder zu falsifizieren: Der Ausdruck »Junggeselle « findet auf vorgestellte, hypothetische oder fiktionale Junggesellen ebenso Anwendung wie auf echte Junggesellen, und somit gibt es keinen Grund, weshalb nicht auch Erstere meine Dispositionen zum Gebrauch von »Junggeselle« auslösen sollten.

Natürlich können wir Fälle konstruieren, in denen Urteile über meinen Sprachgebrauch, die ich aus dem Lehnstuhl fälle, von meiner tatsächlichen Sprachverwendung abweichen. Wir können uns beispielsweise vorstellen, jemand würde mich fragen: »Verwendest du das Wort `Lüge< für alle Fälle, in welchen jemand eine unwahre Behauptung aufstellt? «, und ich würde diese Frage irrtümlicherweise mit »Ja« beantworten. Am nächsten Tag tritt eine Situation auf, in der jemand etwas behauptet, von dem ich weiß, dass es unwahr ist. Andererseits weiß ich aber auch, dass die andere Person ehrlich davon überzeugt ist, dass sie etwas Wahres gesagt hat. Ich werde gefragt, ob die Person gelogen hat, und verneine diese Frage. Der ausschlaggebende Unterschied ist hier aber nicht der zwischen echten und bloß vorgestellten Situationen. Ich hätte auch aus dem Lehnstuhl das korrekte Urteil getroffen, wenn man mich mit einem hypothetischen Fall der Art konfrontiert hätte, wie er am nächsten Tag eingetreten ist. Und solchen Fällen trägt Jackson Rechnung, indem er die oben bereits erwähnte Bedingung aufstellt, dass die relevanten Sprachgebrauchs-Intuitionen einer Diskussion standhalten können müssen. ${ }^{88}$

Wenn wir es mit Ausdrücken wie »Junggeselle« oder auch »Hammer« zu tun haben, für deren Extension in kontrafaktischen Welten die Extension in der aktualen Welt nicht relevant ist, könnte man auf die Idee kommen, dass man die Bedingungen »unter der Annahme, dass die beschriebene Situation in der aktualen Welt eintritt« beziehungsweise »unter der Annahme, dass unsere tatsächliche Welt die aktuale Welt ist« auch einfach weglassen könnte, so dass Dowell überhaupt keinen Anlass dazu hätte, die Frage, ob PseudoÜberzeugungen unsere Dispositionen zuverlässig auslösen oder nicht, zu thematisieren. Diese Idee greift allerdings zu kurz, da wir Fragen der Art (10) und (11) inklusive der jeweiligen Bedingungen bereits benötigen, um feststellen zu können, ob es sich bei einem Ausdruck um einen zweidimensionalen Term 
wie »Wasser« oder einen »eindimensionalen« Term wie »Junggeselle« oder »Hammer« handelt. ${ }^{89}$

Soviel zu dem, was Jackson auf Dowells Einwände entgegnen könnte. Vor dem Hintergrund einer Methode möglicher Fälle, die eher an Hanfling denn an Jackson orientiert ist, beziehungsweise vor dem Hintergrund der Position, die im vorliegenden Buch verteidigt wird, sieht die Sache noch einmal anders aus. Betrachten wir noch einmal die Form der für die Methode möglicher Fälle relevanten Urteile:

(12) Ich würde in der Situation $s$ den Ausdruck $a$ verwenden.

Oder adäquater:

(13) Ich würde über die Situation $s$ sagen, dass sie ein Fall von $F$ ist.

Aussagen der Form (12) oder (13) sind der hier verteidigten Auffassung zufolge keine Hypothesen überVerhaltensdispositionen, sondern sind Ausdruck davon, wie der Sprecher oder die Sprecherin einen bestimmten Ausdruck versteht, und, sofern er oder sie den Ausdruck richtig, d. h. im Einklang mit dem entsprechenden Standard versteht, Ausdruck seiner oder ihrer Sprachkompetenz.

Statt (12) oder (13) könnten wir im Kontext der Beurteilung von Gedankenexperimenten auch Urteile der Form (14) oder (15) verwenden:

(14) In der Situation $s$ ist es meines Erachtens korrekt, den Ausdruck $a$ zu verwenden.

(15) Es entspricht meinem Sprachgefühl, die Situation $s$ als einen Fall von $F$ zu beschreiben..$^{90}$

Es ist zwar richtig, dass unser Sprachgefühl beziehungsweise unsere Sprachkompetenz mit bestimmten Dispositionen einhergehen und dass ich, wenn ich zum Beispiel weiß, dass »Junggeselle unverheirateter Mann bedeutet, auch dazu disponiert sein werde, »Junggeselle « in diesem Sinne zu verwenden. Aber es liegt kein Widerspruch darin zu sagen:

(16) In der Situation $s$ ist es meines Erachtens korrekt, den Ausdruck $a$ zu verwenden. Ich verwende diesen Ausdruck allerdings selber in der Regel nicht, ich verwende eher den synonymen Ausdruck $b$.

Und sogar die folgende Aussage ist konsistent:

(17) In der Situation $s$ ist es meines Erachtens korrekt, den Ausdruck $a$ zu verwenden. Ich habe allerdings ärgerlicherweise die Disposition, in den betreffenden Situationen nicht $a$, sondern den falschen Ausdruck $c$ zu verwenden. Ich sollte mir das unbedingt abgewöhnen.

89 Vgl. Jackson 1998, S. $5^{2}$.

9o Für eine andere sinnvolle Auffassung von Aussagen der Art »Ich würde sagen, dass ...« bzw. »Wir würden sagen, dass ...«, vgl. das Kapitel 7 des vorliegenden Buchs (dort insb. Abschnitt 3.1). 
Andere Dispositionen als die Disposition, einen Ausdruck in bestimmten Situationen zu verwenden, sind enger mit meinem Sprachgefühl verwoben. Insbesondere die Disposition, eine fremde Verwendung des Ausdrucks als richtig oder falsch zu bezeichnen, wenn ich danach gefragt werde. Auch in diesem Zusammenhang ließen sich weit hergeholte Beispiele konstruieren, in welchen meine Dispositionen von meinem Sprachgefühl abweichen: Auch eine solche Abweichung ist im Einzelfall konsistent beschreibbar. Aber im Allgemeinen müssen meine Dispositionen zur Bezeichnung eines Sprachgebrauchs als richtig oder falsch mit meinen Äußerungen der Art von (12) oder (13) übereinstimmen, damit man über mich sagen kann, dass ich diese Äußerungen verstehe.

Darüber, welche Sprachverwendung ich als korrekt erachte beziehungsweise welche Sprachverwendung meinem Sprachgefühl entspricht, habe ich die Autorität der ersten Person. Urteile der Form (12) oder (13) sind mithin Bekenntnisse oder »Avowals « im in der Literatur zu Wittgenstein geprägten Sinn: Die Aufrichtigkeit von Äußerungen solcher Urteile (durch kompetente Sprecherinnen und Sprecher) ist konstitutiv für ihre Wahrheit. Natürlich kann ich mich darüber irren, wie ich sprachliche Ausdrücke tatsächlich verwende, und ich kann auch in einer Diskussion davon überzeugt werden, dass bestimmte Klassifikationen, über die ich zuvor behauptet habe, dass ich sie als korrekt erachte, auf den zweiten Blick nicht mit meinem Sprachgefühl übereinstimmen. Aber erstens zeigt dies nicht, dass mir der Sprachgebrauch, von dem ich behauptet habe, dass er mir korrekt erscheint, nicht im Moment meiner entsprechenden Äußerung tatsächlich korrekt erschienen ist. Und zweitens ist dafür, welche Verwendung ich bei näherer Betrachtung als korrekt erachte, wieder nichts anderes ausschlaggebend, als weitere von mir gefällte Urteile der Form (12) und (13).

Wittgenstein zufolge können wir Bekenntnisse nicht sinnvoll mit »Ich weiß, dass ... « einleiten. ${ }^{91}$ Dies deshalb, weil die Inanspruchnahme von Wissen nur in Kontexten Sinn ergibt, in denen man zum Beispiel auch zweifeln, vermuten oder überzeugt sein kann. ${ }^{92}$ Dies alles ist im Kontext von Bekenntnissen, etwa bei »Ich habe Schmerzen «, nicht möglich: Ich kann nicht daran zweifeln, vermuten oder davon überzeugt sein, dass ich Schmerzen habe. Wenn diese Argumentation stichhaltig ist und es sich bei Urteilen der Form (12) und (13) um Bekenntnisse handelt, ergibt es keinen Sinn, zu fragen, ob solche Urteile

$91 \quad$ Vgl. $P U \S 246, \ddot{U} G \S 41$.

92 Vgl. $P U \S \S 288,408, P P F \S 311, \ddot{U} G \S 58$. Manche von Wittgenstein geprägte Philosophinnen und Philosophen - z. B. Hans-Johann Glock und John Hyman - sind in diesen Punkten allerdings nicht mit Wittgenstein einverstanden. 
a priori oder a posteriori sind. Denn diese Unterscheidung findet nur auf Urteile Anwendung, in Bezug auf die wir Wissen erlangen können.

Man kann die Auffassung, dass »Ich habe Schmerzen«, »Ich erachte diese Beschreibung als korrekt« oder »Diese Formulierung steht im Einklang mit meinem Sprachgefühl« nicht sinnvoll mit »Ich weiß, dass ...« kombinierbar sind, auch ablehnen und trotzdem daran festhalten, dass solche Urteile weder a priori noch a posteriori sind (im gewöhnlichen, nicht in Jacksons Sinn). Auch wenn jemand sagen möchte, er verfüge über Wissen hinsichtlich seiner eigenen Schmerzen, wird er im Idealfall nicht sagen wollen, er verifiziere das Urteil, dass er selbst Schmerzen habe, auf irgendeine Weise. Da die $A$ priori/a posteriori-Unterscheidung auf die kanonische Weise der Verifikation von Urteilen abstellt, wären Urteile der Form (12) und (13) also selbst dann nicht $a$ priori oder a posteriori, wenn wir in Bezug auf sie über Wissen verfügen, und folglich wäre diese Unterscheidung auch nicht auf eine Begriffsanalyse anwendbar, die auf solchen Urteilen basiert.

In den vorangehenden Abschnitten habe ich zunächst Oswald Hanflings und Frank Jacksons Methoden dafür, Begriffe zu analysieren, einander gegenübergestellt. Jackson bezeichnet seine eigene Methode als »Methode möglicher Fälle«. Diese Methode besteht darin, eine Reihe von Fällen durch die Variation verschiedener Parameter zu konstruieren, und dann in Bezug auf die einzelnen Fälle zu entscheiden, ob wir sie als Fälle von Wissen, freiem Willen, Überzeugungen etc. bezeichnen würden (je nachdem, welcher Begriff analysiert werden soll). Der Ausgangspunkt von Hanflings Analyse sind demgegenüber direkt Fälle, in Bezug auf die eindeutig feststeht, dass und wie wir die betreffenden Wörter auf sie anwenden würden - Fälle, die für unseren Gebrauch dieser Ausdrücke charakteristisch sind. Ich habe deshalb vorgeschlagen, Hanflings Methode in Abgrenzung zu Jacksons Methode möglicher Fälle als »Methode typischer Fälle« zu bezeichnen. Wie sich gezeigt hat, unterscheiden sich Jacksons und Hanflings Konzeptionen der Begriffsanalyse auch in weiteren Aspekten, z. B. hinsichtlich der Auffassungen von Begriffen, die die beiden vertreten, und hinsichtlich dessen, wie sie die Relevanz von wissenschaftlichen Theorien für die Festlegung von Begriffen einschätzen.

Im zweiten Teil des Kapitels wurde ein Einwand gegen die Methode möglicher Fälle diskutiert. Dieser Einwand beruht auf der Annahme, dass Urteile der Form »Ich würde diesen Fall als Fall von $F$ (z. B. als Fall von Wissen) bezeichnen«, wie sie bei Jackson (aber auch bei Hanfling) eine wichtige Rolle 
spielen, Hypothesen über sprachliche Dispositionen zum Ausdruck bringen. Wäre dies der Fall, wäre es natürlich problematisch, dass weder Jackson noch Hanfling einräumen, dass Begriffsanalysen prinzipiell durch unsere zukünftige Verwendung sprachlicher Ausdrücke widerlegbar sind. Ich habe dafür argumentiert, dass wir Jackson nicht so verstehen sollten, dass seine Aussagen darüber, wie er bestimmte Wörter verwenden würde, Hypothesen darüber sind, wie er sich unter kontrafaktischen Umständen verhalten würde. Und auch der hier verteidigten Konzeption zufolge sind Aussagen der Form »Ich würde diesen Fall als Fall von $F$ bezeichnen« nicht als Hypothesen über Verhaltensdispositionen aufzufassen, sondern als Bekenntnisse. 